\title{
Medical Prevention of Breast Cancer
}

\author{
Johannes Stubert Max Dieterich Bernd Gerber
}

Department of Obstetrics and Gynecology, University of Rostock, Germany

\section{Keywords}

Breast cancer - Prevention - Tamoxifen - SERMs . Aromatase inhibitors

\section{Summary}

Breast cancer is the most common cancer of women in Western Europe and North America. Effective strategies of medical prevention could reduce the burden of breast cancer mortality. The best evidence for a risk reduction exists for hormonal agents such as tamoxifen and raloxifene $(22-72 \%)$ or aromatase inhibitors (50-65\%). However, the severity of side effects and the lack of evidence for an improved survival compromise the risk/benefit balance. In this review the results of chemoprevention studies, including new treatment approaches, are summarized with critical discussion of their use in clinical practice.

\section{Introduction}

In Germany, women have a lifetime risk for development of breast cancer of about $12 \%$ (70,000 cases per year) and a risk of death following breast cancer of about $3 \%(17,000$ cases per year) [1]. These figures are comparable to those in other developed Western European and North American countries, with breast cancer being the most common cancer among women. Breast cancer is associated not only with an individual patient's suffering, but also with substantial health care cost. Therefore, strategies for prevention could provide both a reduction in mortality and morbidity as well as economic benefits. A distinction is made between different types of prevention depending on the intention [2]. This article deals with primary prevention, which comprises strategies for avoiding the development of a disease a priori, and which is performed on healthy people, usually those who carry an increased risk for disease. Therefore, consideration of the 'primum non nocere' is fundamental. Conflicts with this Hippocratic principle are the main limitation of the known medical agents for breast cancer prevention.

\section{Risk Factors and Models of Risk Assessment}

Identifying women at increased risk for developing breast cancer improves the likelihood of benefit from medical prevention. The greatest risk factor is a germline mutation in the tumor suppressor gene BRCA1 or BRCA2, with an up to $80 \%$ lifetime risk, and preventive strategies for women bearing such mutations will be discussed in a special article in this issue. Beside female sex, the most important populationbased risk factor for breast cancer development is age. According to the Surveillance, Epidemiology, and End Results (SEER) Cancer Statistics Review, the absolute 10-year breast cancer risk increases up to an age of 70 years to a maximum of $3.82 \%$ [3]. The risk declines slightly for older women. The results of the German annual cancer report showed comparable results, with a 10 -year risk maximum of $3.7 \%$ at an age of 65 years [1]. Although the role of sex steroids in the pathogenesis of breast cancer has not yet been fully elucidated, many risk factors are clearly associated with a modulation of the endocrine environment such as reproductive factors, use of oral contraceptives or postmenopausal estrogen-progestin replacement therapy, as well as an increased body mass index [4].

Risk factors such as high breast tissue density, previous premalignant breast lesions and a family history of breast cancer have been associated with a greater increase in relative risk. In a recent meta-analysis, relative risk (adjusted for age) in women with high breast density of $\geq 75 \%$ was 4.20 (95\%

\section{KARGER \\ Fax +497614520714

\section{(c) 2014 S. Karger AG, Freiburg}

1661-3791/14/0096-0391\$39.50/0
Dr. med. Johannes Stubert

Department of Obstetrics and Gynecology

University of Rostock

Südring 81, 18059 Rostock, Germany

johannes.stubert@uni-rostock.de 
confidence interval (CI) 3.61-4.89) compared to women with a density $<5 \%[5,6]$. Relative risk increases remarkably if first-degree relatives (mother, sister) have a history of breast cancer [7-9].

An increased breast cancer risk is also evident if a prior breast biopsy revealed an atypical ductal hyperplasia (odds ratio (OR) 2.4 ; 95\% CI 1.3-4.5) or atypical lobular hyperplasia (OR 5.3; 95\% CI 2.7-10.4) [10]. A subgroup of individuals with high breast cancer risk comprises women who received radiation to the chest region during childhood following malignant diseases (e.g. Hodgkin's lymphoma). They showed an increase in the cumulative breast cancer incidence of $30 \%$ (95\% CI 25-34) at age 50 [11] and standardized incidence ratios between 21.9 and 24.7 [11, 12].

Modifiable risk factors (use of hormone therapy, obesity, breast feeding) directly offer the possibility of being optimized to give a reduction in breast cancer risk. Although many risk factors are non-modifiable (e.g. breast density, menarche, ethnicity, relatives with breast cancer), they can be used for an individual risk assessment, facilitating a decision for or against a chemopreventive treatment.

\section{Models of Risk Assessment}

Various risk stratification models allow an estimation of breast cancer risk over a specific period by integrating a number of risk factors [13]. The Gail model - the first major risk model - and its modified version (after integration of SEER data) was used in prevention studies for individual risk calculation (see table 1, online supplemental material; www.karger. com/?DOI=369573). Subsequent models (e.g. Claus, Rosner and Colditz, Barlow, Tice or Cuzick and Tyrer) used a similar approach, varying in their reference standards and the risk modifying factors [13]. In a systematic review, most models demonstrated a high calibration (that means high concordance between predicted and observed breast cancer risk in a population), but the discriminatory accuracy (which gives information on how correctly an individual person was classified at higher risk) was low to modest (c-statistics $0.55-0.65$; 0.5 means the model performs no better than chance) in predicting individual breast cancer probability [13].

\section{Risk Reduction by Selective Estrogen Receptor Modulators}

There is strong evidence that the use of selective estrogen receptor modulators (SERMs; tamoxifen, raloxifene etc.) can lead to a reduction of the risk for breast cancer (table 1, online supplemental material; www.karger.com/? DOI=369573). The preventive activity of tamoxifen was demonstrated in several randomized controlled trials, showing a hazard ratio (HR) for breast cancer development between 0.84 and 0.57
[14-21]. The effect was mainly determined by a reduction of endocrine-sensitive breast cancers. In an updated meta-analysis of the placebo-controlled trials tamoxifen nearly halved the risk $(44 \%$ reduction, $\mathrm{p}<0.001)$ in this subgroup [22]. The incidence of ductal carcinoma in situ (DCIS) was also reduced (HR 0.72; 95\% CI 0.57-0.92), but the frequency of estrogen receptor-negative breast cancers was non-significantly increased (HR 1.13; 95\% CI 0.86-1.49). Similar results were achieved if other SERMS such as raloxifene [23-26] or the third-generation substances lasofoxifene and arzoxifene were used [27-30]. In the meta-analysis [22], overall reduction in breast cancer (including DCIS) was 38\% ( $<<0.0001)$ for all SERMs. The estimated 10-year cumulative incidence was $6.3 \%$ in the placebo-treated group and $4.2 \%$ in the SERM group, with a number-needed-to-treat of 42 women to prevent 1 case of breast cancer [22]. Although the mean treatment duration was only 4 years, the protective effect persisted for 5-10 years [22]. Even though smaller, the carryover effect was significant. In contrast to the other SERMs, raloxifene did not influence the frequency of DCIS [22]. In a head-to-head comparison tamoxifen was more effective than raloxifene (RR 1.24 ; $95 \%$ CI 1.05-1.47) in reducing the incidence of invasive breast cancer [31]. According to the MORE trial, 93 women would need to be treated with raloxifene for 4 years to avoid 1 case of invasive breast cancer [24, 25].

Preparations of black cohosh (Cimicifuga racemosa) contain phytoestrogens with purported SERM-like activity, and are widely used for the management of menopausal symptoms; 1 study reported a risk reduction for breast cancer development in postmenopausal users (OR 0.47; 95\% CI 0.27 0.82 ) [32]. However, the findings of the retrospective analyses were inconsistent, and the results were not confirmed by others [33].

None of the placebo-controlled studies revealed any evidence that SERMs could induce a reduction of breast cancerrelated mortality. In the NSABP-P1 trial, the largest study that included only patients at increased risk for breast cancer, no difference in breast cancer-related survival was observed [15]. Further interpretation of the study results was hindered as, after stopping the recruitment, the patients of the placebo group were offered the use of tamoxifen. In the IBIS-1-trial, which had the longest follow-up of 96 months, no risk reduction for breast cancer-related death was found (RR 0.85; 95\% CI 0.34-2.05), and the number of deaths from any cause tended to be higher in the tamoxifen group than in the placebo group (RR 1.18; 95\% CI 0.81-1.73) [21]. Moreover, a meta-analysis that included the 9 placebo-controlled SERM trials did not found any advantage of SERM treatment in reducing mortality from any cause (HR 0.98; 95\% CI 0.90-1.06) or from breast cancer (HR 1.03, 95\% CI 0.55-1.92) [22].

\section{Harm and Additional Benefits of SERMs}

Harm caused by SERM treatment limits the general preventive use. Venous thromboembolic events, including life- 
threatening pulmonary embolism, and an increase of endometrial cancer are the most relevant forms of harm associated with SERM use. The risk of deep venous thrombotic events was shown to be increased in all studies, with an overall OR of 1.73 (95\% CI 1.47-2.05) without differences between tamoxifen and raloxifene. The rate seemed to be even higher for arzoxifene and lasofoxifene. Highest risk for thromboembolic events was observed in women older than 50 years [34].

Overall, no negative effect of SERMs was found for cardio- and neurovascular events, but the incidence of fatal stroke was increased in the raloxifene arm of the RUTH trial (HR 1.49; 95\% CI 1.00-2.24) [22, 23]. In this study the cardiovascular protective effect of raloxifene was the primary study endpoint, and only patients at increased cardiovascular risk were included. The results suggest a critical use of SERMs in this subgroup of patients.

With the exception of raloxifene, all of the investigated SERMs increased the risk of endometrial cancer (HR 1.56; 95\% CI 1.13-2.14) [22]. The risk of endometrial cancer was 3 times higher in the tamoxifen group compared to that in the placebo group in the NSABP-P1 trial (RR 3.28; 95\% CI 1.87$6.03)$ [15]. In the IBIS-1 trial, the majority of cases with endometrial cancer were FIGO (International Federation of Gynecology and Obstetrics) stage I, and the risk was highest in women at age of 50 years or older [20,21]. As raloxifene did not increase the risk of endometrial cancer, this is an option for women who have not been hysterectomized [24, 25, 31].

The increased risks of venous thromboembolic events and of endometrial cancer were only apparent during the time of treatment without carryover effect [22]. All SERMs had a positive effect on bone density with a reduction of osteoporotic fractures.

\section{Risk Reduction by Aromatase Inhibitors}

The preventive effect of aromatase inhibitors on breast cancer risk was studied in 2 prospective randomized, placebocontrolled trials (table 1, online supplemental material; $w w w$. karger.com/? DOI=369573). Risk reduction for invasive breast cancer was $65 \%$ with exemestane in the MAP.3 trial, and 50\% with anastrozole in the IBISII trial $[35,36]$. Like SERMs, the aromatase inhibitors only reduced the incidence of estrogen receptor-positive cancer. The incidence of DCIS was also significantly reduced In the IBIS II trial (HR 0.30; 95\% CI 0.12 0.74) [36], but not in the exemestane trial [35]. According to the 7-year follow-up, to prevent 1 invasive breast cancer, 36 women would need to be treated with anastrozole. The number of women needed-to-treat was 94 in 3 years and 26 in 5 years in the MAP. 3 trial. Breast cancer-related mortality as well as total death rates did not differ between treatment and placebo in either trial.

\section{Harm and Additional Benefits of Aromatase Inhibitors}

The aromatase inhibitors did not influence the risk of thromboembolic events or endometrial cancer [35, 36]. No increase of osteoporotic events was observed, suggesting that the differences between SERMs and aromatase inhibitors in the large adjuvant trials were mainly a result of the bone protective effect of tamoxifen $[37,38]$. However, with aromatase inhibitors, an increased bone loss was demonstrated by bone density measurements in the MAP.3 trial despite calcium and vitamin D supplementation [39]. Osteoporosis following the suppression of estrogen levels is, therefore, the most profound side effect of the aromatase inhibitors. Musculoskeletal (11$64 \%$ ) and vasomotoric symptoms (40-57\%) were significantly more frequent in the aromatase inhibitor arms, and may lower a patient's compliance $[35,36]$. However, it was notable that patients in the placebo group also frequently complained about these symptoms (vasomotoric symptoms in $32-49 \%$ ) $[35,36]$. In the IBISII trial, more cases of hypertension (RR 1.64; $95 \%$ CI 1.18-2.28) and of dry eyes (RR 1.45; 95\% CI 1.04-2.01) were found, but the MAP.3 trial did not confirm these. Surprisingly, the rate of colorectal cancers (RR 0.28; 95\% CI 0.08-0.99) was lower despite estrogen depression in the IBISII trial. Again, this was not observed in the MAP.3 trial.

\section{Potential Non-Endocrine Active Agents for Medical Prevention}

The development of agents with preventive activity on hormone receptor-negative breast cancer is mandatory. Presently, no substance has proved adequate for a use outside of studies, but several potentially chemopreventive agents are still currently under investigation.

\section{Metformin}

In a recent meta-analysis, reviewing 7 observational studies, the use of metformin was associated with a lower breast cancer incidence (OR 0.83; 95\% CI 0.71-0.97) in diabetic women [40]. Preclinical studies suggested a preventive effect on breast cancer development [41]. Moreover, metformin use for 2 weeks in a neoadjuvant setting was associated with a decrease of tumor cell proliferation [42, 43], although the effect was not observed in all studies [44]. Several clinical trials are in progress to investigate the breast cancer preventive activity of metformin [45].

\section{Retinoids}

The vitamin A analogues regulate gene expression, and several retinoids have demonstrated cancer preventive effects in preclinical breast cancer models [45]. The less-toxic synthetic analog fenretinide has been already investigated in human studies. In a long-term follow-up of a phase III trial, fenretinide significantly reduced the risk of secondary breast 
cancer development in premenopausal women (HR 0.62; $95 \%$ CI 0.46-0.83) [46, 47]. No preventive effect was observed in postmenopausal women [46]. The preventive activity of fenretinide in premenopausal women was confirmed by a further placebo-controlled randomized trial (HR 0.38; 95\% CI 0.15$0.90)$ [48]. The small study $(n=235)$ included women with early breast cancer or an intraepithelial neoplasia in their history and women with increased breast cancer risk according to the Gail model. Interestingly, a combination of tamoxifen with fenretinide did not show any preventive activity. 'Rexinoids' such as bexarotene, LG100268 and 9cUAB30 are new synthetic retinoic acid derivatives that only activate retinoid $\mathrm{X}$ receptors (RXR). Preclinical studies suggest a preventive potential on hormone receptor-negative breast cancer [45].

\section{Statins}

Data on the preventive activity of statins for reduction of breast cancer risk are controversial. A meta-analysis including 24 observational studies with more than 2.4 million participants did not support the hypothesis of a preventive effect (RR 0.99; 95\% CI 0.94-1.04) [49]. Similar results were reported for a second meta-analysis including 7 randomized trials and 9 observational studies (RR 1.03; 95\% CI 0.93-1.14) [50].

\section{NSAIDs, COX-2 Inhibitors and Aspirin}

Analysis of randomized controlled trials of the use of aspirin for protection of cardiovascular events revealed a reduction of breast cancer risk [51]. A meta-analysis of nonsteroidal anti-inflammatory drugs (NSAID) including aspirin and ibuprofen revealed a relative risk of 0.88 (95\% CI 0.84-0.93) for breast cancer development [52]. Use of aspirin, but not of other NSAIDs, was also associated with reduced breast cancer risk (RR 0.80; 95\% CI 0.71-0.90) in a population based cohort analysis of 26,580 postmenopausal women [53]. In this study, risk reduction did not differ according to hormone receptor status. However, a recent prospective cohort analysis only found a modest non-significant reduce of breast cancer risk (RR 0.91; 95\% CI 0.81-1.01) with regular aspirin use in postmenopausal women [54]. Risk reduction was independent of hormone receptor status, and did not differ in respect to the molecular breast cancer subtype. Meanwhile, a phase III study investigating the efficacy of the COX2 inhibitor celecoxib on breast cancer recurrence, the REACT trial, is underway.

\section{Bisphosphonates}

Two cohort analyses demonstrated an association between bisphosphonate use and a decreased breast cancer incidence $[55,56]$. Postmenopausal women of the Women's Health Initiative (WHI) who used oral bisphosphonates presented with lower invasive breast cancer frequency (HR 0.68; 95\% CI $0.52-0.88$ ) [55]. Risk reduction was independent of hormone receptor status; however, due to the low absolute number of cases, the results for the hormone receptor negative group were not significant. Interpretation of the study findings was hampered by differences between the characteristics of patients in bisphosphonate user and non-user groups, but generally, according to Gail model, users of bisphosphonates were initially at increased breast cancer risk compared to non-users. In a population-based case-control study of Israeli patients with breast cancer, the use of bisphosphonates for longer than 1 year before breast cancer diagnosis also was associated with decreased relative risk of breast cancer (OR 0.72; 95\% CI 0.57-0.90) and tumors developing under bisphosphonate use tended to have a favorable prognostic profile [56]. Again, there were significant differences in patients' characteristics between cases and controls, but results remained significant after adjustment for several risk factors. The majority of patients used oral bisphosphonates. These results were supported by a British cohort analysis (HR 0.71, 95\% CI 0.62-0.81) and a British nested case-control study (OR 0.92; 95\% CI 0.87-0.97) - both found a decreased breast cancer risk after bisphosphonate use [57, 58]. However, the relation between low estrogen levels, decreased bone mass and breast cancer risk remains unclear, and prospective trials investigating the chemopreventive impact of bisphosphonates still underway.

\section{Others}

Several other agents targeting diverse molecular pathways (e.g. epidermal growth factor receptor (EGFR)-tyrosine kinase, poly (ADP-ribose) polymerase (PARP), hypoxia inducible factor (HIF), PI3K-mTOR, nuclear factor (NF)- $\mathrm{kB}$, insulin-like growth factor (IGF)) were estimated to have preventive activity on breast cancer. As some of these agents only develop anti-tumor activity in breast cancer subgroups (e.g. PARP inhibitors in cells with loss of BRCA function, HER2 targeting substances), their potential preventive effect may also be restricted to special high-risk groups (e.g. patients with familiar BRCA mutation, patients with DCIS). However, most of the new agents have not been yet proven in a clinical setting on humans. Data have been summarized in several instructive reviews $[45,59,60]$.

\section{Discussion}

With respect to the randomized controlled trials of chemoprevention using SERMs or aromatase inhibitors, there is no doubt about the efficacy of these agents in reducing the risk of breast cancer. However, the studies could not determine whether this risk reduction will also lead to an improved survival. Follow-up and study populations were insufficient to answer this important question; however, even a meta-analysis including more than 80,000 women was not able to find a trend towards improved survival [22]. Even assuming a reduction of breast cancer-related deaths, agents like tamoxifen 
may (at least in part) antagonize this gain by its life-threatening side effects such as pulmonary embolism or endometrial cancer. As most of the prevented breast cancers are small at time point of detection (due to screening mammography) and of low aggressive biology (mostly hormone receptor-positive and node-negative), the prognosis of these tumors is exceptionally good and most of the women will be cured in this situation. Consequently, if any real benefit is to be expected from the use of the endocrine prevention, the identification of patients at high risk will be necessary. With the exception of the STAR trial, all raloxifene studies included patients without breast cancer risk estimation and the primary study endpoint was not the development of breast cancer. Moreover, the estimated 5-year breast cancer risk of $1.66 \%$ according to the Gail model seemed to be too low, and greatest risk reduction was achieved in women with a risk of $>5 \%$ or a history of atypical hyperplasia or lobular carcinoma in situ [13]. The efficiency of an endocrine prevention in cases of BRCA mutation and a history of familial breast cancer remains uncertain.

Recommended treatment duration for SERMs is 5 years [61], although information about adherence (constancy of dose and interval) and persistence (duration of therapy) are limited [13]. In the Italian Tamoxifen Prevention study, only $60.8 \%$ of patients completed the 5 -year treatment period and, on average, treatment duration was 4.0 years $[16,17]$. The results are representative for the other trials that have reported a treatment compliance between $60 \%$ and $80 \%$ [13].

In conclusion, we know the effects of SERMs and aromatase inhibitors in breast cancer prevention, but we do not know which group of women would benefit most from preventive therapy. Furthermore, even with preventive treatment it remains uncertain whether therapy will improve survival, and if so, how long a patient should be treated. Although tamoxifen and raloxifene have been approved for breast cancer prevention treatment by the American Food and Drug Asso- ciation (FDA), the treatment recommendation has the codicil '...should be discussed as an option...' [61]. The German Arbeitsgemeinschaft Gynäkologische Onkologie (AGO) suggests a chemopreventive treatment only as an individualized approach in patients at increased risk and after extensive consulting [62]. In Europe none of these agents has been approved by the European Medicines Agency (EMA), and so any use would be off-label. Stringent patient selection (exclusion of thromboembolic risk factors, avoidance of tamoxifen in women who have not been hysterectomized) may help to reduce harm caused by treatment. Although aromatase inhibitors have not yet been approved by the FDA for breast cancer prevention, the revised guidelines of the American Society of Clinical Oncology (ASCO) as well as the AGO open a treatment option for postmenopausal women. However, unresolved problems are similar to those for SERMs and side effects may also hamper the acceptance of aromatase inhibitors. The new study findings of the MAP. 3 and IBISII trials may lead to only limited progress in practice [63]. The development of strategies for risk reduction of hormone receptornegative breast cancer may increase the impact of preventive therapies in high-risk patients.

\section{Supplemental Material}

Table 1. Summary of randomized controlled studies for medical breast cancer prevention

To access the supplemental table, please refer to $w w w$. karger.com/?DOI=369573.

\section{Disclosure Statement}

The authors reported no conflicts of interest.

\section{References}

1 Kaatsch P, Spix C, Hentschel S, et al.: Krebs in Deutschland 2009/2010. 9. Ausgabe. Berlin, 2013.

$\checkmark 2$ Slesina W: Primordial, primary, secondary and tertiary prevention. A definition. Dtsch Med Wochenschr 2007;132:2196-2198.

3 Howlader N, Noone AM, Krapcho M, et al.: SEER Cancer Statistics Review, 1975-2009, National Cancer Institute. Bethesda, MD (http://seer.cancer. gov/csr/1975_2011/).

4 Hankinson SE, Eliassen AH: Circulating sex steroids and breast cancer risk in premenopausal women. Horm Cancer 2010;1:2-10.

$\checkmark 5$ Cummings SR, Tice JA, Bauer S, et al.: Prevention of breast cancer in postmenopausal women: Approaches to estimating and reducing risk. J Natl Cancer Inst 2009;101:384-398.

6 Schreer I: Dense breast tissue as an important risk factor for breast cancer and implications for early detection. Breast Care (Basel) 2009;4:89-92.
7 Collaborative Group on Hormonal Factors in Breast Cancer: Familial breast cancer: Collaborative reanalysis of individual data from 52 epidemiological studies including 58,209 women with breast cancer and 101,986 women without the disease. Lancet 2001;358:1389-1399.

8 Colditz GA, Rosner BA, Speizer FE: Risk factors for breast cancer according to family history of breast cancer. For the Nurses' Health Study Research Group. J Natl Cancer Inst 1996;88:365-371.

9 Colditz GA, Kaphingst KA, Hankinson SE, Rosner B: Family history and risk of breast cancer: nurses' health study. Breast Cancer Res Treat 2012;133:1097-1104.

10 Marshall LM, Hunter DJ, Connolly JL, et al.: Risk of breast cancer associated with atypical hyperplasia of lobular and ductal types. Cancer Epidemiol Biomarkers Prev 1997;6:297-301.
1 Moskowitz CS, Chou JF, Wolden SL, et al.: Breast cancer after chest radiation therapy for childhood cancer. J Clin Oncol 2014;32:2217-2223.

12 Kenney LB, Yasui Y, Inskip PD, et al.: Breast cancer after childhood cancer: A report from the Childhood Cancer Survivor Study. Ann Intern Med 2004;141:590-597.

13 Nelson HD, Smith MEB, Griffin JC, Fu R: Use of medications to reduce risk for primary breast cancer: A systematic review for the U.S. Preventive Services Task Force. Ann Intern Med 2013;158: 604-614.

14 Fisher B, Costantino JP, Wickerham DL, et al.: Tamoxifen for prevention of breast cancer: report of the National Surgical Adjuvant Breast and Bowel Project P-1 Study. J Natl Cancer Inst 1998; 90:1371-1388. 
15 Fisher B, Costantino JP, Wickerham DL, et al.: Tamoxifen for the prevention of breast cancer: Current status of the National Surgical Adjuvant Breast and Bowel Project P-1 study. J Natl Cancer Inst 2005;97:1652-1662.

16 Veronesi U, Maisonneuve P, Rotmensz N, et al.: Tamoxifen for the prevention of breast cancer: Late results of the Italian Randomized Tamoxifen Prevention Trial among women with hysterectomy. J Natl Cancer Inst 2007;99:727-737.

17 Veronesi U, Maisonneuve P, Costa A, et al.: Prevention of breast cancer with tamoxifen: Preliminary findings from the Italian randomised trial among hysterectomised women. Italian Tamoxifen Prevention Study. Lancet 1998;352:93-97.

18 Powles T, Eeles R, Ashley S, et al.: Interim analysis of the incidence of breast cancer in the Royal Marsden Hospital tamoxifen randomised chemoprevention trial. Lancet 1998;352:98-101.

19 Powles TJ, Ashley S, Tidy A, et al.: Twenty-year follow-up of the Royal Marsden randomized, double-blinded tamoxifen breast cancer prevention trial. J Natl Cancer Inst 2007;99:283-290.

20 Cuzick J, Forbes J, Edwards R, et al.: First results from the International Breast Cancer Intervention Study (IBIS-I): A randomised prevention trial. Lancet 2002;360:817-824.

21 Cuzick J, Forbes JF, Sestak I, et al.: Long-term results of tamoxifen prophylaxis for breast cancer 96-month follow-up of the randomized IBIS-I trial J Natl Cancer Inst 2007;99:272-282.

22 Cuzick J, Sestak I, Bonanni B, et al.: Selective oestrogen receptor modulators in prevention of breast cancer: An updated meta-analysis of individual participant data. Lancet 2013;381:1827-1834.

23 Barrett-Connor E, Mosca L, Collins P, et al.: Effects of raloxifene on cardiovascular events and breast cancer in postmenopausal women. N Engl J Med 2006;355:125-137.

24 Cauley JA, Norton L, Lippman ME, et al.: Continued breast cancer risk reduction in postmenopausal women treated with raloxifene: 4-year results from the MORE trial. Multiple outcomes of raloxifene evaluation. Breast Cancer Res Treat 2001;65:125134.

25 Cummings SR, Eckert S, Krueger KA, et al.: The effect of raloxifene on risk of breast cancer in postmenopausal women: Results from the MORE randomized trial. Multiple Outcomes of Raloxifene Evaluation. JAMA 1999;281:2189-2197.

26 Martino S, Cauley JA, Barrett-Connor E, et al.: Continuing outcomes relevant to Evista: Breast cancer incidence in postmenopausal osteoporotic women in a randomized trial of raloxifene. J Natl Cancer Inst 2004;96:1751-1761.

27 Cummings SR, Ensrud K, Delmas PD, et al.: Lasofoxifene in postmenopausal women with osteoporosis. N Engl J Med 2010;362:686-696.

28 LaCroix AZ, Powles T, Osborne CK, et al.: Breast cancer incidence in the randomized PEARL trial of lasofoxifene in postmenopausal osteoporotic women. J Natl Cancer Inst 2010;102:1706-1715.

29 Powles TJ, Diem SJ, Fabian CJ, et al.: Breast cancer incidence in postmenopausal women with osteoporosis or low bone mass using arzoxifene. Breast Cancer Res Treat 2012;134:299-306.

30 Cummings SR, McClung M, Reginster J-Y, et al.: Arzoxifene for prevention of fractures and invasive breast cancer in postmenopausal women. J Bone Miner Res 2011;26:397-404.
31 Vogel VG, Costantino JP, Wickerham DL, et al.: Update of the National Surgical Adjuvant Breast and Bowel Project Study of Tamoxifen and Raloxifene (STAR) P-2 Trial: Preventing breast cancer. Cancer Prev Res (Phila) 2010;3:696-706.

32 Rebbeck TR, Troxel AB, Norman S, et al.: A retrospective case-control study of the use of hormonerelated supplements and association with breast cancer. Int J Cancer J Int Cancer 2007;120:1523-1528.

33 Fritz H, Seely D, McGowan J, et al.: Black cohosh and breast cancer: a systematic review. Integr Cancer Ther 2014;13:12-29.

34 Iqbal J, Ginsburg OM, Wijeratne TD, et al.: Endometrial cancer and venous thromboembolism in women under age 50 who take tamoxifen for prevention of breast cancer: A systematic review. Cancer Treat Rev 2012;38:318-328.

35 Goss PE, Ingle JN, Alés-Martínez JE, et al.: Exemestane for breast-cancer prevention in postmenopausal women. N Engl J Med 2011;364:2381-2391.

36 Cuzick J, Sestak I, Forbes JF, et al.: Anastrozole for prevention of breast cancer in high-risk postmenopausal women (IBIS-II): An international, double-blind, randomised placebo-controlled trial. Lancet 2014;383:1041-1048.

37 Perez EA, Josse RG, Pritchard KI, et al.: Effect of letrozole versus placebo on bone mineral density in women with primary breast cancer completing 5 or more years of adjuvant tamoxifen: A companion study to NCIC CTG MA.17. J Clin Oncol 2006;24: 3629-3635.

38 Eastell R, Adams JE, Coleman RE, et al.: Effect of anastrozole on bone mineral density: 5-year results from the anastrozole, tamoxifen, alone or in combination trial 18233230. J Clin Oncol 2008;26:10511057.

39 Cheung AM, Tile L, Cardew S, et al.: Bone density and structure in healthy postmenopausal women treated with exemestane for the primary prevention of breast cancer: A nested substudy of the MAP.3 randomised controlled trial. Lancet Oncol 2012;13:275-284.

$40 \mathrm{Col}$ NF, Ochs L, Springmann V, et al.: Metformin and breast cancer risk: A meta-analysis and critical literature review. Breast Cancer Res Treat 2012; 135:639-646.

41 Thompson AM: Molecular pathways: Preclinical models and clinical trials with metformin in breast cancer. Clin Cancer Res 2014;20:2508-2515.

42 Hadad S, Iwamoto T, Jordan L, et al.: Evidence for biological effects of metformin in operable breast cancer: A pre-operative, window-of-opportunity, randomized trial. Breast Cancer Res Treat 2011; 128:783-794.

43 Niraula S, Dowling RJO, Ennis M, et al.: Metformin in early breast cancer: A prospective window of opportunity neoadjuvant study. Breast Cancer Res Treat 2012;135:821-830.

44 Bonanni B, Puntoni M, Cazzaniga M, et al.: Dual effect of metformin on breast cancer proliferation in a randomized presurgical trial. J Clin Oncol 2012;30:2593-2600.

45 Litzenburger BC, Brown PH: Advances in preventive therapy for estrogen-receptor-negative breast cancer. Curr Breast Cancer Rep 2014;6:96-109.

46 Veronesi U, Mariani L, Decensi A, et al.: Fifteenyear results of a randomized phase III trial of fenretinide to prevent second breast cancer. Ann Oncol 2006;17:1065-1071.
47 Veronesi U, De Palo G, Marubini E, et al.: Randomized trial of fenretinide to prevent second breast malignancy in women with early breast cancer. J Natl Cancer Inst 1999;91:1847-1856.

48 Decensi A, Robertson C, Guerrieri-Gonzaga A, et al.: Randomized double-blind $2 \times 2$ trial of lowdose tamoxifen and fenretinide for breast cancer prevention in high-risk premenopausal women. $\mathrm{J}$ Clin Oncol 2009:27:3749-3756.

49 Undela K, Srikanth V, Bansal D: Statin use and risk of breast cancer: A meta-analysis of observational studies. Breast Cancer Res Treat 2012;135: 261-269.

50 Bonovas S, Filioussi K, Tsavaris N, Sitaras NM: Use of statins and breast cancer: A meta-analysis of seven randomized clinical trials and nine observational studies. J Clin Oncol 2005 1;23:8606-8612.

51 Rothwell PM, Price JF, Fowkes FGR, et al.: Shortterm effects of daily aspirin on cancer incidence, mortality, and non-vascular death: analysis of the time course of risks and benefits in 51 randomised controlled trials. Lancet 20128;379:1602-1612.

52 Takkouche B, Regueira-Méndez C, Etminan M: Breast cancer and use of nonsteroidal anti-inflammatory drugs: a meta-analysis. J Natl Cancer Inst 2008;100:1439-1447.

53 Bardia A, Olson JE, Vachon CM, et al.: Effect of aspirin and other NSAIDs on postmenopausal breast cancer incidence by hormone receptor status: results from a prospective cohort study. Breast Cancer Res Treat 2011;126:149-155.

54 Zhang X, Smith-Warner SA, Collins LC, et al.: Use of aspirin, other nonsteroidal anti-inflammatory drugs, and acetaminophen and postmenopausal breast cancer incidence. J Clin Oncoll 2012;30 3468-3477.

55 Chlebowski RT, Chen Z, Cauley JA, et al.: Oral bisphosphonate use and breast cancer incidence in postmenopausal women. J Clin Oncol 2010;28: 3582-3590.

56 Rennert G, Pinchev M, Rennert HS: Use of bisphosphonates and risk of postmenopausal breast cancer. J Clin Oncol 2010;28:3577-3581.

57 Cardwell CR, Abnet CC, Veal P, et al.: Exposure to oral bisphosphonates and risk of cancer. Int J Cancer 2012;131:E717-725

58 Vinogradova Y, Coupland C, Hippisley-Cox J: Exposure to bisphosphonates and risk of common non-gastrointestinal cancers: Series of nested casecontrol studies using two primary-care databases. Br J Cancer 2013 6;109:795-806.

59 Den Hollander P, Savage MI, Brown PH: Targeted therapy for breast cancer prevention. Front Oncol 2013;3:250.

60 Cazzaniga M, Bonanni B: Breast cancer chemoprevention: Old and new approaches. J Biomed Biotechnol 2012;2012:985620.

61 Visvanathan K, Hurley P, Bantug E, et al.: Use of pharmacologic interventions for breast cancer risk reduction: American Society of Clinical Oncology clinical practice guideline. J Clin Oncol 20130;31: 2942-2962.

62 Arbeitsgemeinschaft Gynäkologische Onkologie: (cited 2014 July 29);Available from: www.agoonline.de/fileadmin/downloads/leitlinien/mamma/ maerz2014/de/2014D_02_Brustkrebsrisiko_und_ Praevention.pdf.

63 Cameron DA: Breast cancer chemoprevention: Little progress in practice? Lancet 2014;383:10181020 . 